\title{
Distributed Strain Measurement along a Concrete Beam via Stimulated Brillouin Scattering in Optical Fibers
}

\author{
Romeo Bernini, ${ }^{1}$ Aldo Minardo, ${ }^{2}$ Stefano Ciaramella, ${ }^{3}$ \\ Vincenzo Minutolo, ${ }^{4}$ and Luigi Zeni ${ }^{5}$ \\ ${ }^{1}$ Istituto per Il Rilevamento Elettromagnetico Dell Ambiente, Consiglio Nazionale Delle Ricerche, Naples, Italy \\ ${ }^{2}$ Department of Information Engineering, Second University of Naples, 81031 Aversa (CE), Italy \\ ${ }^{3}$ Dipartimento di Ingegneria Civile, Seconda Università di Napoli, Via Roma, 2981031 Aversa, Italy \\ ${ }^{4}$ Seconda Università di Napoli, Via Roma, 2981031 Aversa, Italy \\ ${ }^{5}$ Istituto Nazionale per la Fisica della Materia, Trieste, Italy \\ Correspondence should be addressed to Aldo Minardo, aldo.minardo@unina2.it
}

Received 18 January 2011; Accepted 25 February 2011

Academic Editor: Francesco Soldovieri

Copyright () 2011 Romeo Bernini et al. This is an open access article distributed under the Creative Commons Attribution License, which permits unrestricted use, distribution, and reproduction in any medium, provided the original work is properly cited.

The structural strain measurement of tension and compression in a $4 \mathrm{~m}$ long concrete beam was demonstrated with a distributed fiber-optic sensor portable system based on Brillouin scattering. Strain measurements provided by the fiber-optic sensor permitted to detect the formation of a crack in the beam resulting from the external applied load. The sensor system is valuable for structural monitoring applications, enabling the long-term performance and health of structures to be efficiently monitored.

\section{Introduction}

Structural health monitoring systems have the potential to reduce operational maintenance costs by identifying problems at an early stage, and to verify the effectiveness of repair procedures. Moreover, monitoring systems help increase understanding of the real behavior of a structure, such as a bridge, and aid in planning maintenance interventions. In the long term, static monitoring requires an accurate and very stable system, which can relate deformation measurements taken over long periods of time [1]. Strain measurement with a distributed Brillouin scattering-based sensor system provides excellent opportunity for the health monitoring of civil structures [2-4]. It allows measurements to be taken along the entire length of the fiber, rather than at discrete points, by using fiber itself as the sensing medium.

Distributed optical fiber sensors based on stimulated Brillouin scattering (SBS) rely on the interaction between two lightwaves and an acoustic wave in the optical fiber. The measurement principle is based on the characteristic that the Brillouin frequency of the optical fiber is shifted when strain as well as temperature changes occur. Spatial information along the length of the fiber can be obtained through Brillouin optical time domain analysis (BOTDA) by measuring propagation times for light pulses travelling in the fiber. This allows for continuous distributions of the measurand to be monitored. This type of sensing has tremendous potential for structural monitoring. These systems offer unmatched flexibility of measurement locations and the ability to monitor a virtually unlimited number of locations simultaneously.

In this paper, we report a number of experimental measurements carried out along a $4 \mathrm{~m}$-long concrete beam subjected to a variable load, by the use of a portable BOTDA sensor. A single-mode optical fiber was attached to the beam in order to detect both tensile and compressive strains. Two different adhesives were employed, in order to compare them as regards their efficiency in transferring the strain between the structure and the fiber core. The sensor was able to detect the formation of a crack in the midsection of the beam, consequent to the application of an external load.

\section{Experimental Results}

BOTDA measurements were carried out by using a portable prototype integrating all the optoelectronic equipment 


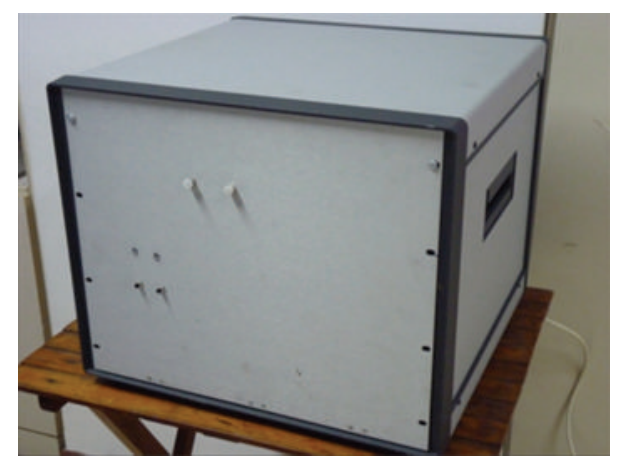

Figure 1: Portable prototype for SBS-based distributed strain and temperature measurements.

necessary to perform distributed strain and temperature measurements in optical fibers. Details on the arrangement implemented can be found in [4]. Here, we focus on the characteristics of the realized prototype, a picture of which is shown in Figure 1. The main features of the instrument are, a relatively short acquisition time, a fully automatic operation and a high signal-to-noise $(\mathrm{S} / \mathrm{N})$ ratio of the measurements. An acquisition time in the order of about one minute was obtained by employing for data acquisition an analog-todigital (A/D) converter with a field-programmable gate array (FPGA) device on the same board. The FPGA can process the output of the A/D converter in real time, we use it to perform an averaging on board of the acquired traces. This permitted to strongly reduce the aggregate data rate and therefore the latency time due to transfer of data to PC. A valuable consequence is that a high number of averages, and therefore a high $\mathrm{S} / \mathrm{N}$ ratio can be obtained while keeping a short acquisition time. Both instrument control and data processing are performed by a central processing unit (CPU) mounted on a mini-ITX board placed internally. External communication with the instrument is realized by setting up a remote desktop control. The optical fiber used for sensing is connected to the prototype optical circuit by two FC adapters located in the front panel of the instrument. Dedicated software was developed by which the whole measuring process as well as post processing of the acquired data are fully automatic. The different measurement parameters can be easily set, and the results of the measurements are visualized and stored. The nominal resolution of the sensor is $\pm 1^{\circ} \mathrm{C}$ for temperature sensing, and $\pm 20 \mu \varepsilon$ for strain sensing.

The laboratory tests described hereafter have been performed using a $4 \mathrm{~m}$-long " $\mathrm{T}$ " cross section pretensed concrete beam. Figure 2 shows a sketch of the beam, together with the positions where the sensing fiber was attached. The sensing fiber was a commercial standard single-mode fiber for telecommunication with polyvinyl chloride $900 \mu \mathrm{m}$ tight-buffer outer jacket. Note that a single optical fiber was bonded four times along the structure along different longitudinal directions. In this way, the strain profiles were acquired simultaneously at each measurement. Two different adhesives were employed for comparison purposes: an epoxy resin for the leftmost positions (A and B), and a polyurethane-based adhesive for the rightmost positions
(C and D). A dial strain gauge was placed at the point of maximum deflection (midsection) in order to validate the measurements provided by the optical fiber sensor. Before application of external loads to the beam, a reference strain profile was acquired by the prototype. This reference profile was employed to subtract the strains induced by the bonding procedure from the successive measurements.

The beam was loaded by using a single-span arrangement and applying different weights at the center of the span. We applied an increasing weight from $50 \mathrm{daN}$ to $200 \mathrm{daN}$ at a step of 25 daN. External temperature was verified to keep constant during the experimental tests. Figure 3 reports a summary of the measurements performed during the whole loading cycle. In particular, Figure 3(a) reports the strains acquired along the two fibers bonded with epoxy resin (positions A and B), while Figure 3(b) refers to the fibers bonded with polyurethane adhesive (positions $\mathrm{C}$ and $\mathrm{D}$ ). For each case, the positive (tensile) strains are relative to the lower fibers ( $\mathrm{B}$ and $\mathrm{D})$, while the negative (compressive) strains refer to the upper fibers (A and C). At first, we note that although the theory predicts a sharp turn of the strain profiles in the midsection, the measured distributions appear smoothened around their maximum. This has to be attributed to the limited spatial resolution of our optical sensor $(1 \mathrm{~m})$, as well as to the limited spatial sampling $(20 \mathrm{~cm})$. Note also that for each applied load the peak tensile strain is always lower than the maximum compressive strain. This was expected as it is directly related to the position at which the fibers were attached with respect to the neutral axis of the structure (see Figure 2(b)). Comparing Figures 3(a) and 3(b), we note that the fibers bonded with epoxy and subjected to tensile strain provide more regular strain profiles as compared to the fibers bonded with polyurethane, that is, the strain distributions are closer to the theoretical profiles. However, the absolute strain levels are highly comparable for the two adhesives. At the condition of maximum load ( $200 \mathrm{daN})$, a crack opened at the midsection of the beam. The effect of the crack on the strain profiles acquired by the optical fibers can be observed in Figure 3, showing a sudden increase in the maximum strain in the concrete when the crack develops.

The beam was then subjected to a progressively decreasing load. Figures 4 and 5 summarize the results of this new measurement cycle. In particular, Figure 4 refers to the fibers bonded with epoxy, while Figure 5 refers to the fibers bonded with polyurethane. Comparing the results with those obtained before crack formation, an increase of strain all over the loaded beam is clearly observable, such an increase being higher in the middle portion of the beam.

For example, referring to the fibers bonded with epoxy, the maximum strain measured at position $B$ with an applied load of $150 \mathrm{daN}$ increases from $\approx 125 \mu \varepsilon$ to $\approx 210 \mu \varepsilon$ after crack formation. Similarly, at position, A the compressive strain at the midsection increases from $\approx 195 \mu \varepsilon$ to $\approx 300 \mu \varepsilon$. Note that, at zero load, a residual tensile strain upto $100 \mu \varepsilon$ and a compressive strain upto $30 \mu \varepsilon$ are measured by the two epoxy-glued fibers.

In order to show the effect of crack opening in a different perspective, we report in Figure 6 the maximum strain 


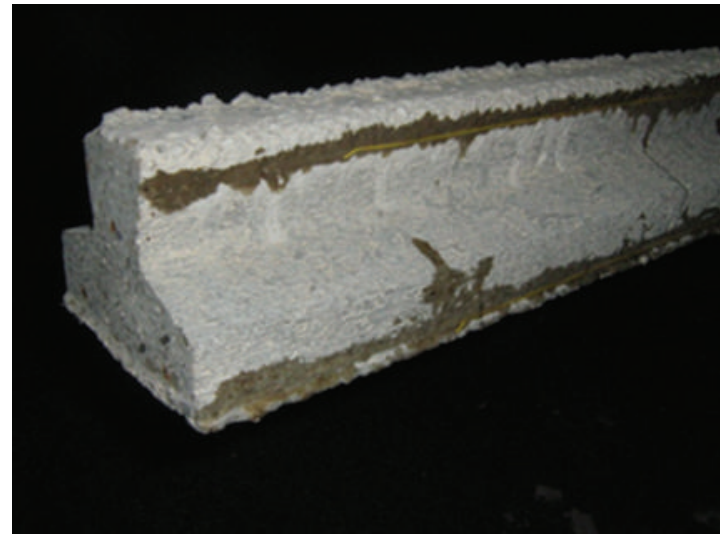

(a)

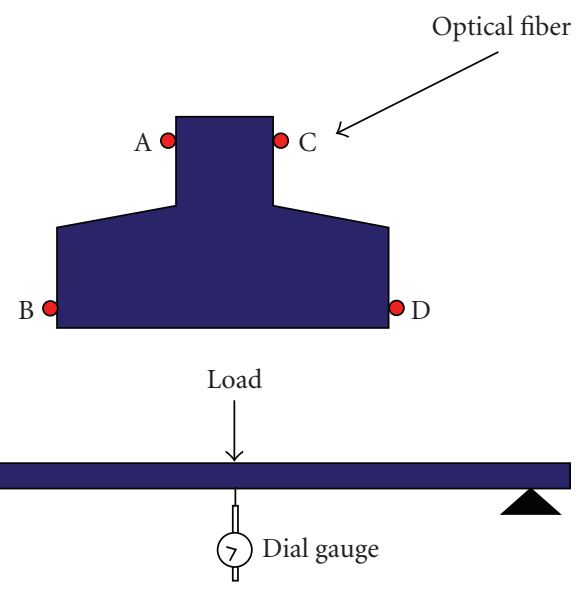

(b)

FIGURE 2: (a) Picture of the concrete beam used for the tests; (b) the crosssection and longitudinal view of the concrete beam used for the tests.

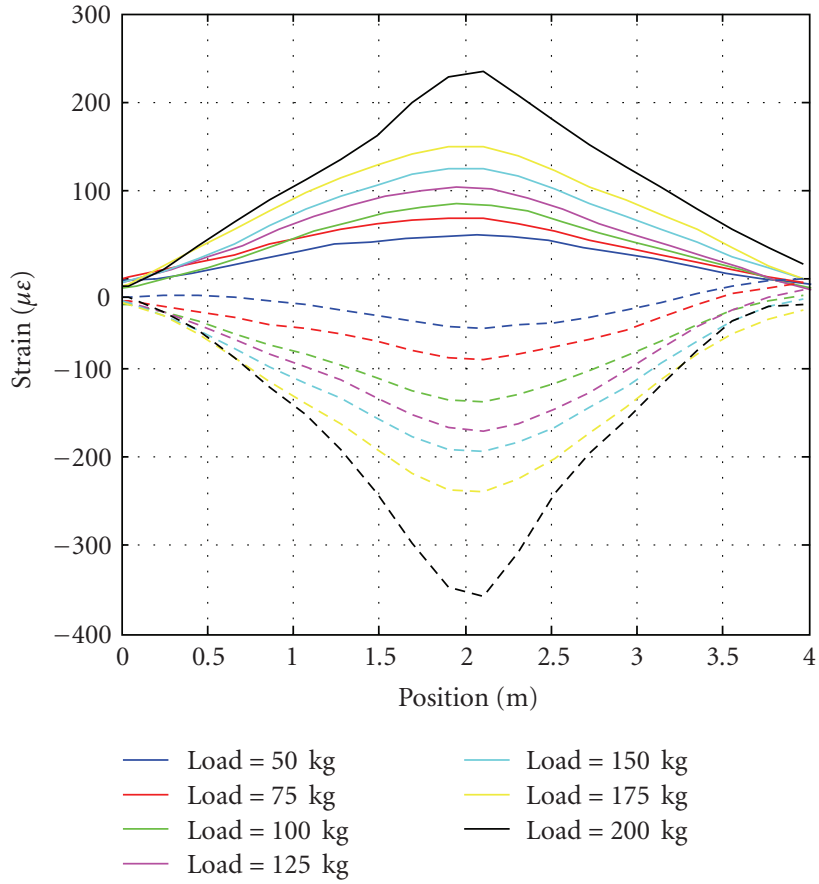

(a)

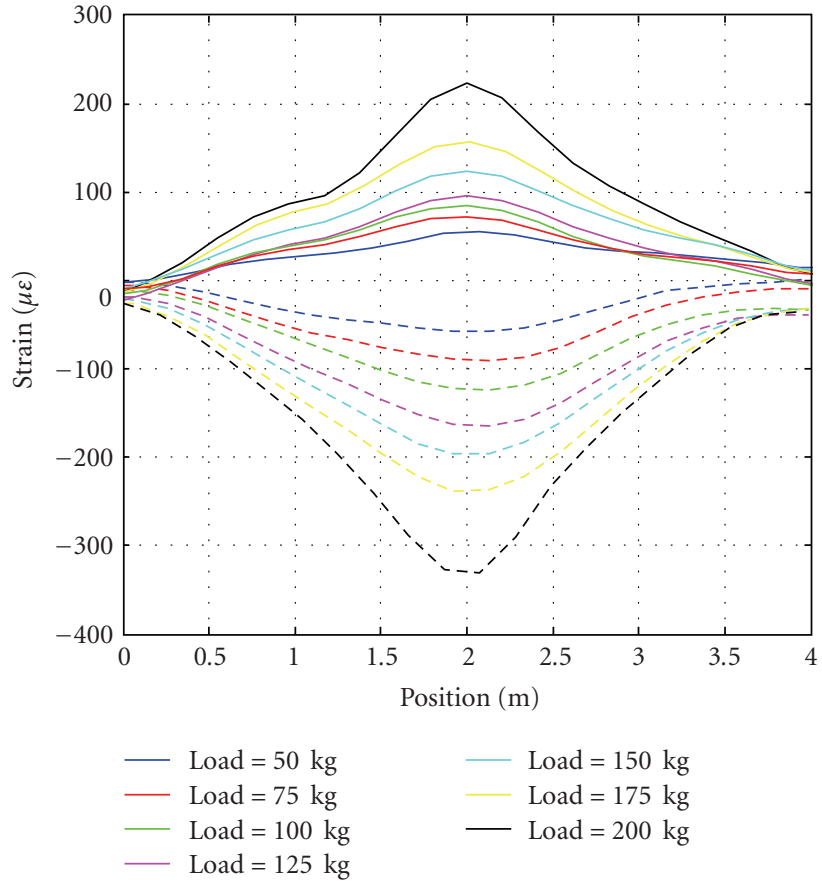

(b)

Figure 3: (a) Measured strain distribution along the length of the beam with fibers bonded with epoxy resin; (b) measured strain distribution along the length of the beam with fibers bonded with polyurethane.

measured during the first (loading) and second (unloading) cycles, for each monitored position and applied weight. It can be seen that, during the first cycle, the strain change increases abruptly when increasing the load from 175 daN to $200 \mathrm{daN}$. Also, during the unloading cycle, the maximum strain is always higher than the corresponding value measured before crack formation.

The crack formation produces an eigenstrain distribution in the cross section of the beam that is revealed by the residual strain values attained at complete unloading. The residual strain distribution gives origin to a localized inelastic curvature given by

$$
\chi_{a}=\frac{\varepsilon_{l}-\varepsilon_{u}}{h}
$$

where $\varepsilon_{l}$ and $\varepsilon_{u}$ are the strains measured at two different quotes distant $h$. We can use the curvature in order to compare data provided by the dial strain gauge, with data provided by the optical fiber sensors. In particular, we use (1) in order to calculate the curvature provided by the two 


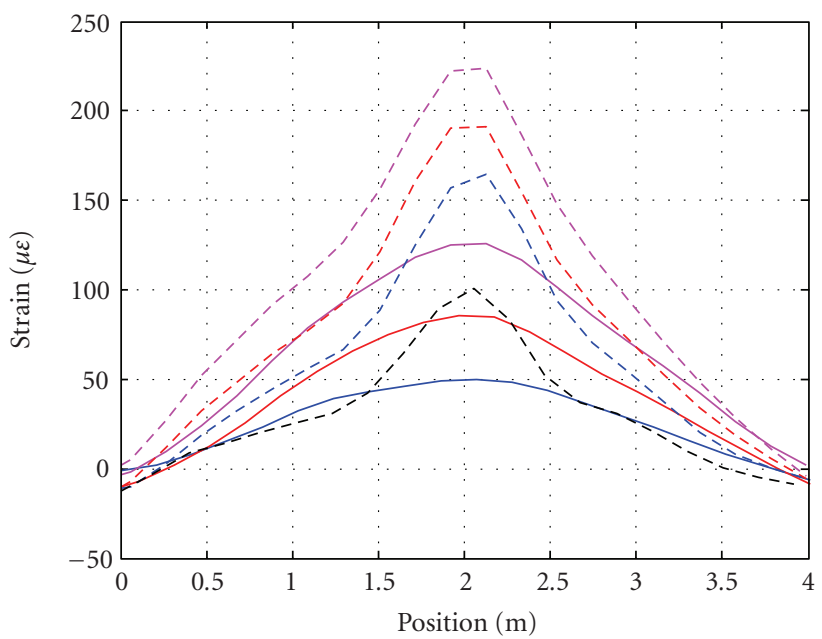

(a)

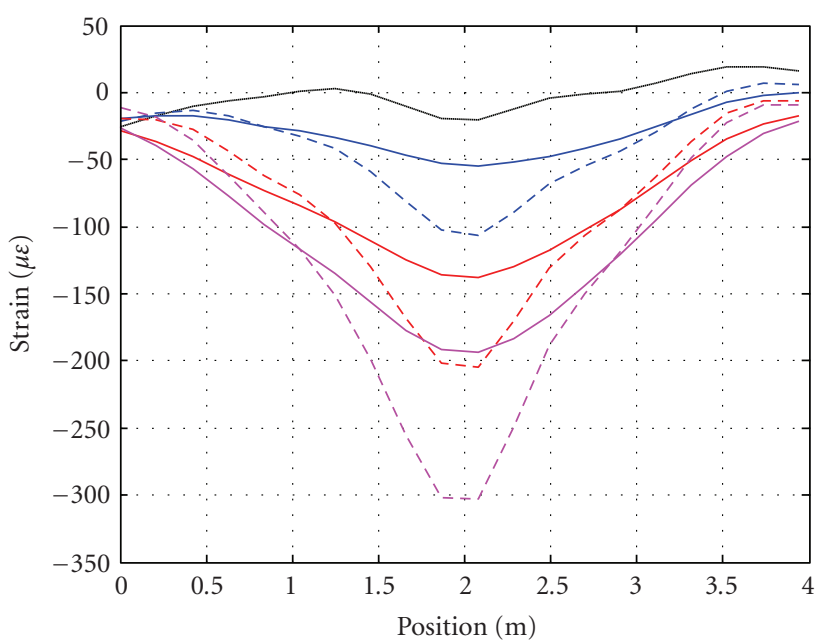

(b)

FIGURE 4: (a) Measured strain distribution along the length of the beam with fiber bonded with epoxy resin (position B); (b) measured strain distribution along the length of the beam with fiber bonded with epoxy resin (position A). Solid line = loading step. Dashed line $=$ unloading step. Blue line $=50 \mathrm{daN}$. Red line $=100 \mathrm{daN}$. Magenta line $=150 \mathrm{daN}$. Black line $=0 \mathrm{daN}$.

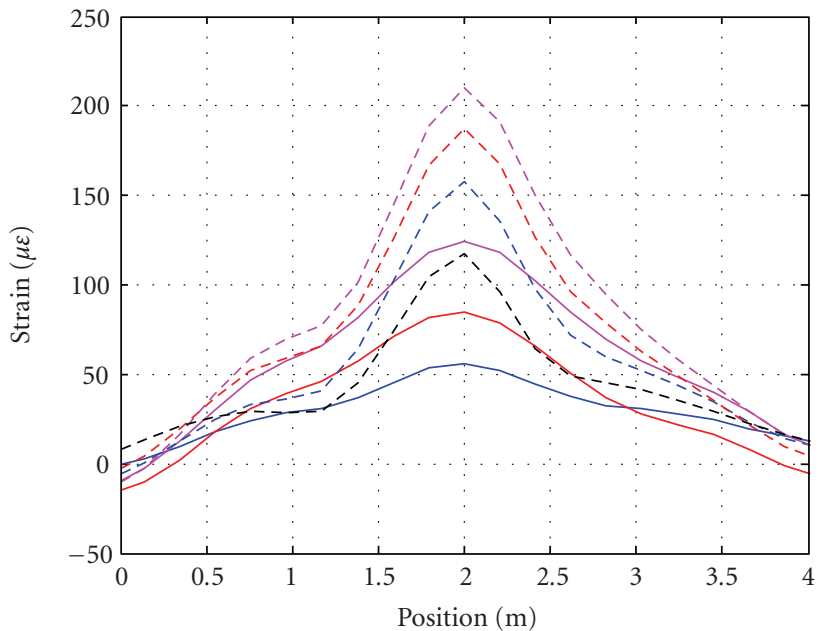

(a)

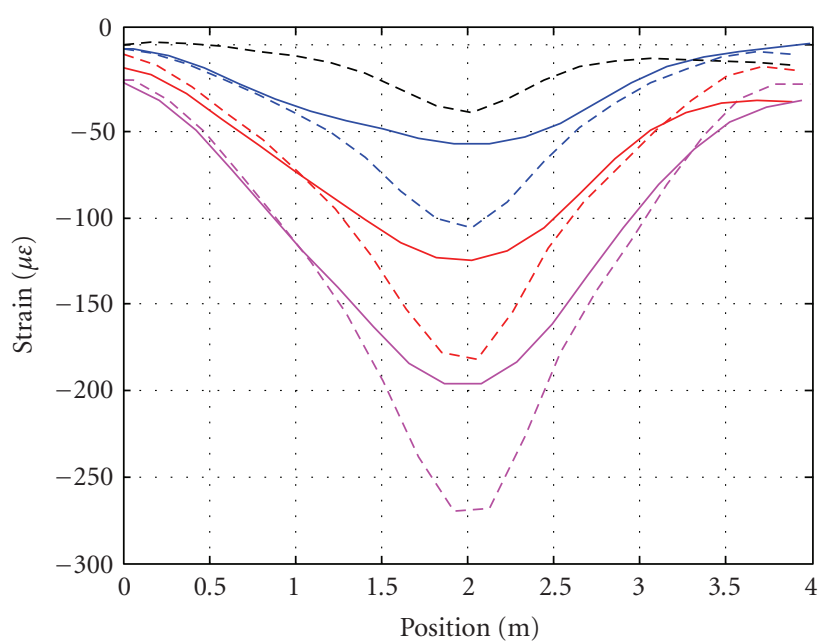

(b)

FIGURE 5: (a) Measured strain distribution along the length of the beam with fiber bonded with polyurethane (position D); (b) measured strain distribution along the length of the beam with fiber bonded with polyurethane (position C). Solid line = loading step. Dashed line $=$ unloading step. Blue line $=50 \mathrm{daN}$. Red line $=100 \mathrm{daN}$. Magenta line $=150 \mathrm{daN}$. Black line $=0 \mathrm{daN}$.

sensors, using a height $h$ of $10 \mathrm{~cm}$ for the dial gauge case, or $h=8.1 \mathrm{~cm}$ (i.e., the vertical distance between fibers A and B, or C and D) for the optical fiber case.

The results are shown in Figure 7. It is seen that a good agreement exists between the curvatures provided by the sensors, except from the measurements carried out after crack formation. The discrepancy can be explained by considering that the optical fiber sensor has a spatial resolution of $\approx 1 \mathrm{~m}$, so the highly localized strains consequent to crack opening are spatially averaged. Therefore, the resulting curvature provided by the optical fiber sensor in the midsection is lower than the actual one, after crack formation. This limitation can be overcome either by the use of a sophisticated signal processing of data, permitting to detect more accurately the crack-induced strains, [5], or by the use of an SBS sensor with improved spatial resolution. A cm scale spatial resolution has the clear advantage of permitting a precise localization of the crack within the monitored structure [6]. Work is in progress in order to push the spatial resolution of the portable instrument in the $\mathrm{cm}$-range.

\section{Conclusions}

A portable distributed Brillouin scattering sensor has been used for structural health monitoring on a concrete beam. The experiments show the correlation of the distributed measured strain with local measurements provided by a dial strain gauge. It has been shown that the formation of a crack 


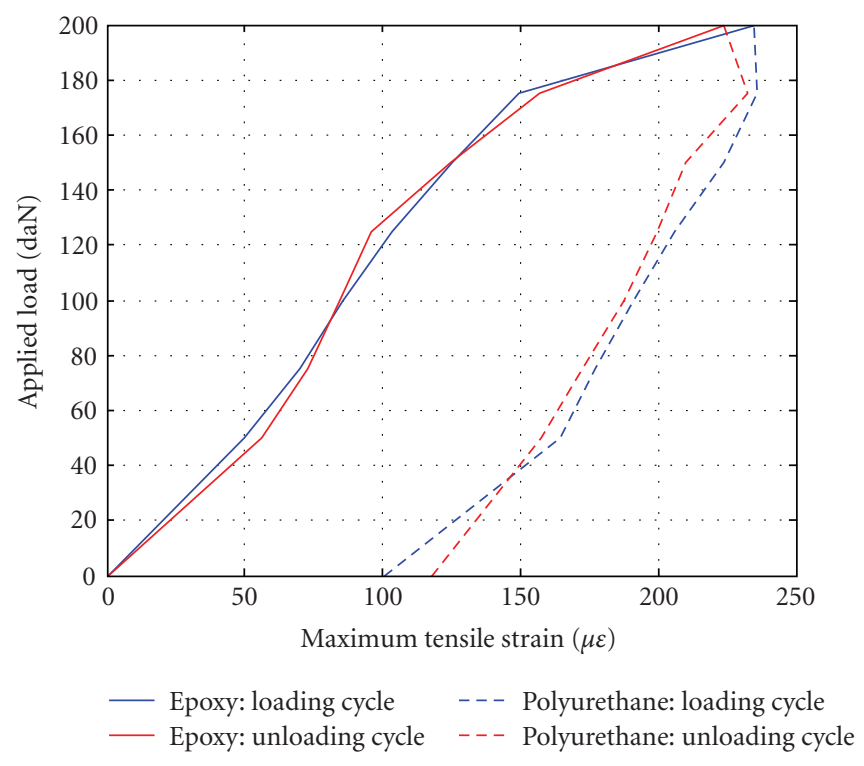

(a)

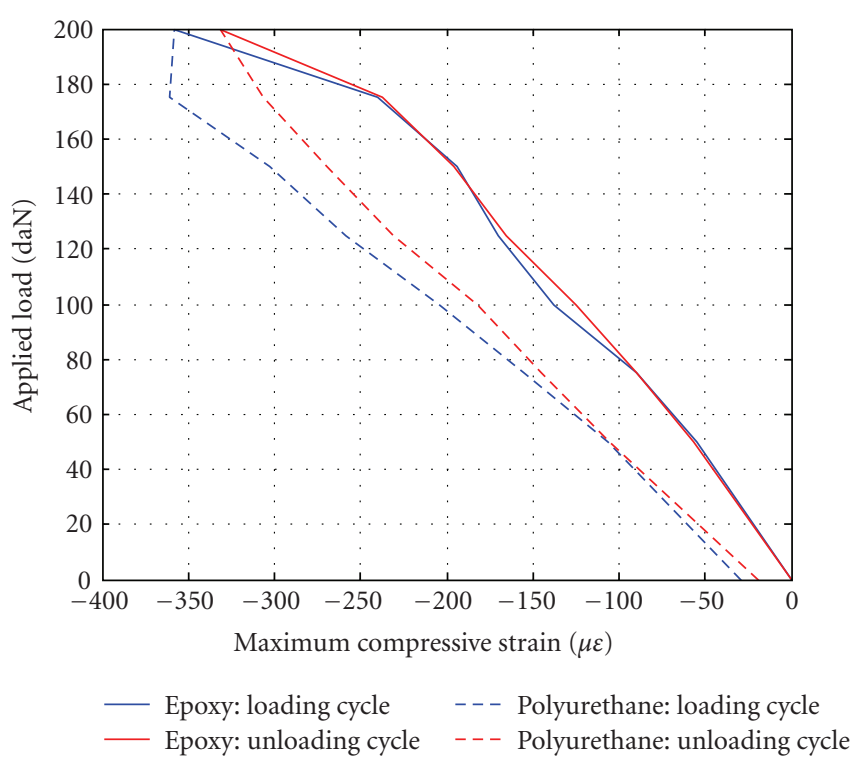

(b)

Figure 6: (a) Applied load versus maximum tensile strain; (b) applied load versus maximum compressive strain.

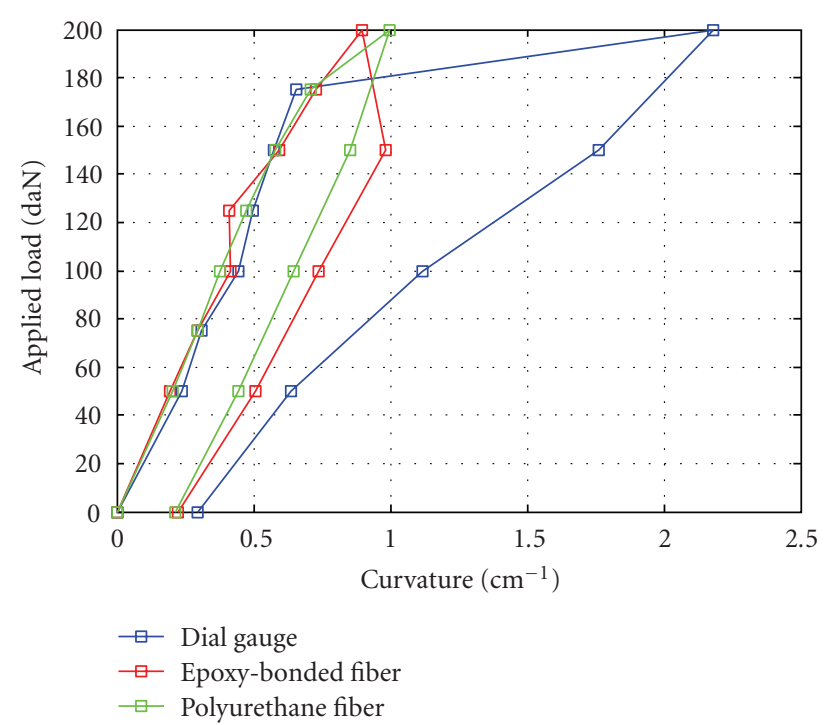

Figure 7: Applied load versus curvature. The latter was calculated at the midsection using the dial strain gauge or the optical fiber sensor measurements.

emerges as an increase of the beam curvature, successfully detected by means of the optical fiber sensor readings.

\section{Acknowledgments}

The research leading to these results has received funding from the European Community's Seventh Framework Programme (FP7/2007-2013) under Grant Agreement no. 225663 Joint Call FP7-ICT-SEC-2007-1 and from Second University of Naples_-PRIST 2009 Project.

\section{References}

[1] O. Burdet, "Automatic deflection and temperature monitoring of a balanced cantilever concrete bridge," in Proceedings of The 5th International Conference of Short and Medium Concrete Bridges., Calgary, Alberta, Canada, July 1998.

[2] T. Horiguchi, K. Shimizu, T. Kurashima, M. Tateda, and Y. Koyamada, "Development of a distributed sensing technique using Brillouin scattering," Journal of Lightwave Technology, vol. 13, no. 7, pp. 1296-1302, 1995.

[3] M. DeMerchant, A. Brown, X. Bao, and T. Bremner, "Structural monitoring by use of a Brillouin distributed sensor," Applied Optics, vol. 38, no. 13, pp. 2755-2759, 1999.

[4] R. Bernini, M. Fraldi, A. Minardo et al., "Identification of defects and strain error estimating in bending steel beams through time-domain Brillouin distributed Optical Fiber Sensors," Smart Materials and Structures, vol. 15, pp. 612-622, 2006.

[5] F. Ravet, F. Briffod, B. Glisic, M. Nikles, and D. Inaudi, "Detection of sub-millimeter faults with a time domain distributed Brillouin sensor," in 19th International Conference on Optical Fibre Sensors, D. Sampson, S. Collins, K. Oh, and R. Yamauchi, Eds., vol. 7004 of Proceedings of SPIE, pp. 1-4, 2008, 700412.

[6] M. Imai, R. Nakano, T. Kono, T. Ichinomiya, S. Miura, and M. Mure, "Crack detection application for fiber reinforced concrete using BOCDA-based optical fiber strain sensor," Journal of Structural Engineering, vol. 136, no. 8, pp. 1001-1008, 2010. 

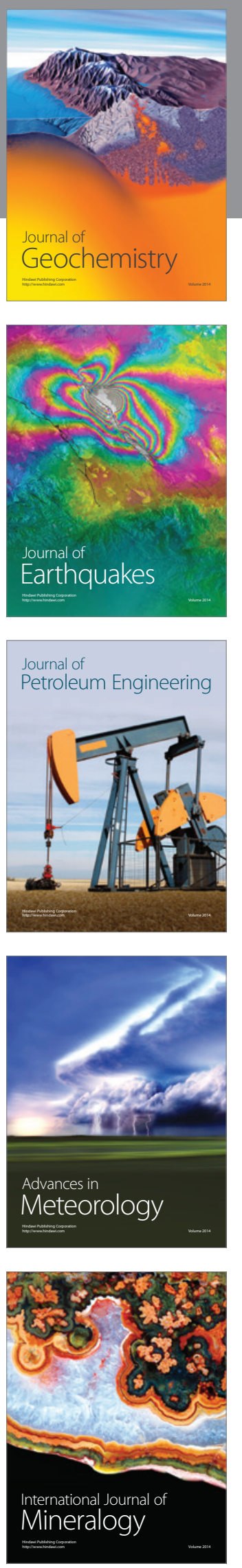
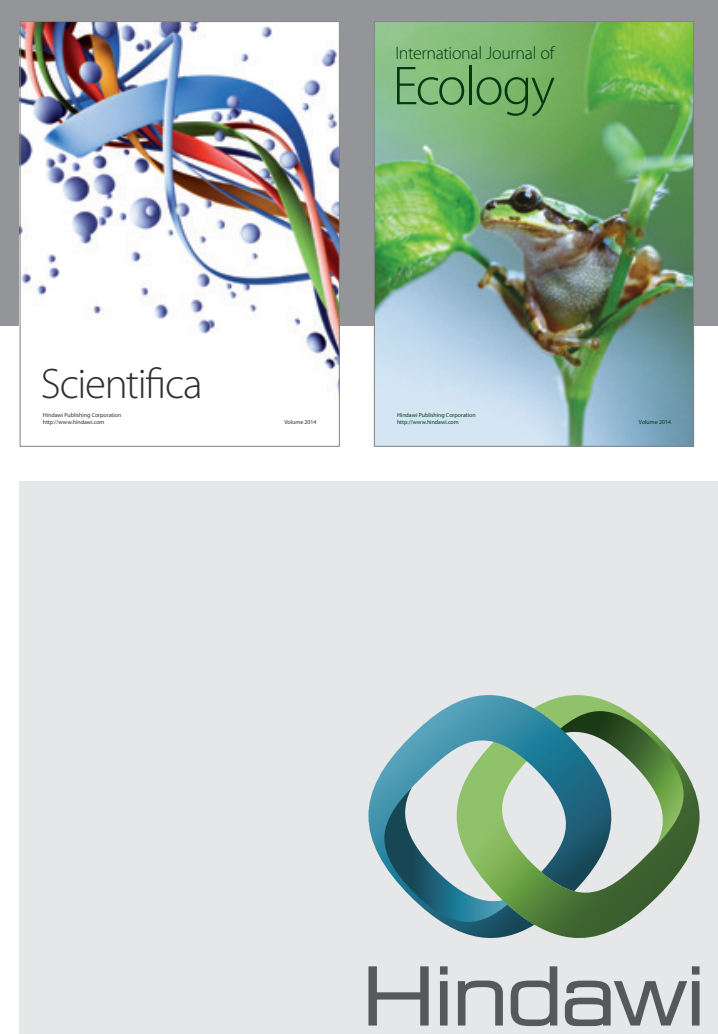

Submit your manuscripts at http://www.hindawi.com
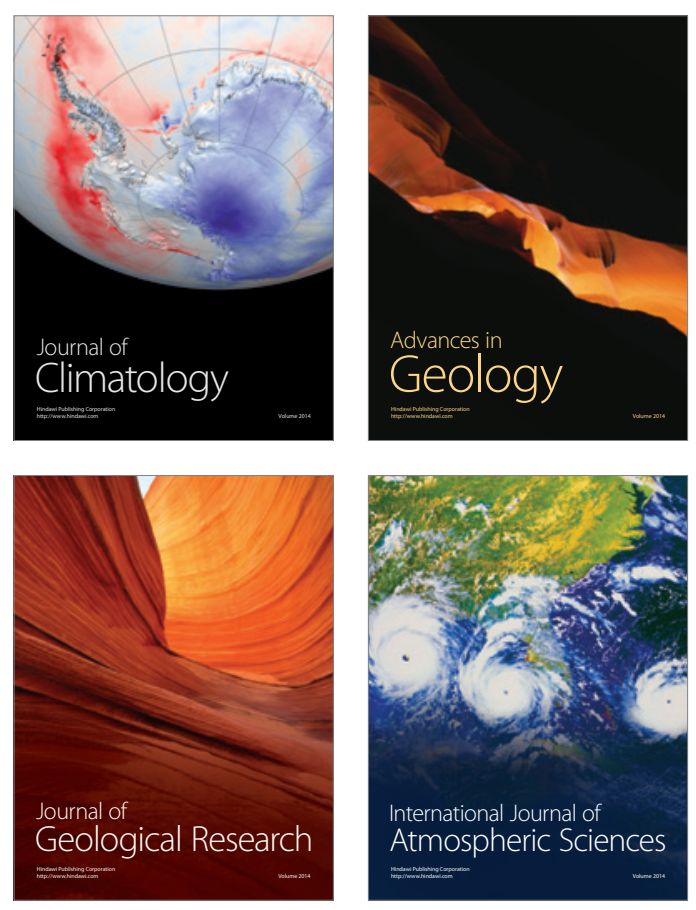
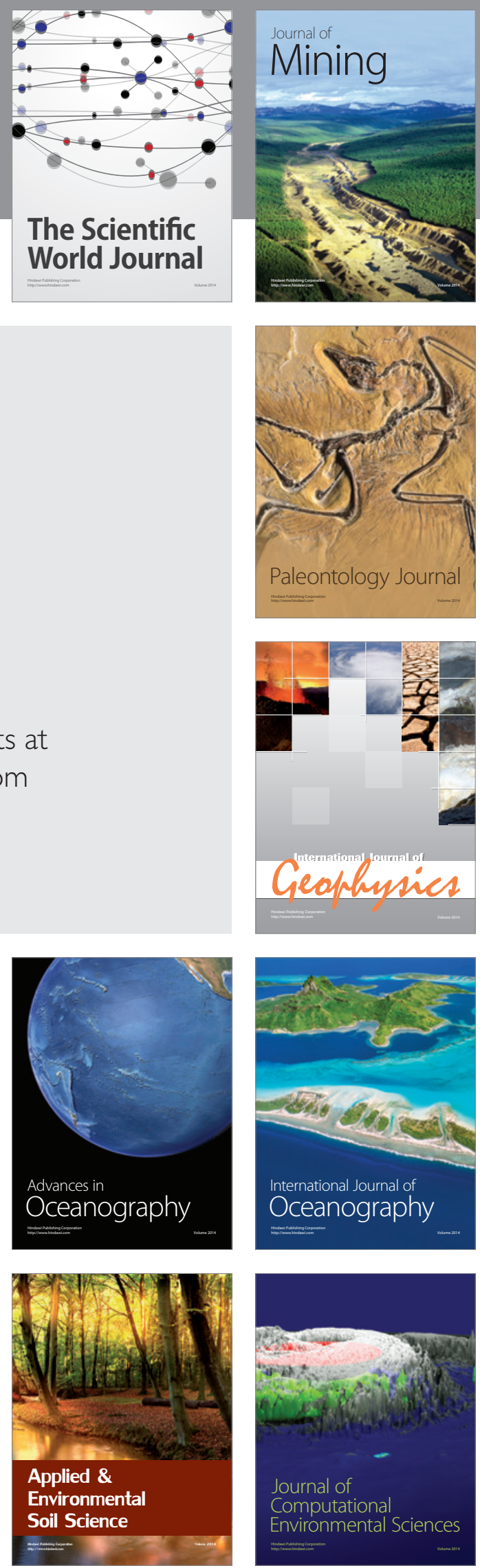